\title{
The order of nilpotence of Hecke operators mod 2: a new proof
}

\author{
Mathilde Gerbelli-Gauthier
}

\section{Correspondence:}

mathilde@math.uchicago.edu Department of Mathematics, The University of Chicago, 5734 S University Ave, 60637 Chicago, IL, USA

\section{照 Springer}

\begin{abstract}
Let $\mathbb{F}_{2}[\Delta]$ be the ring of modular forms of level 1, mod 2, where the coefficients of $\Delta$ are the reduction mod 2 of Ramanujan's $\tau$ function. The Hecke operators act nilpotently on $\mathbb{F}_{2}[\Delta]$. The order of nilpotence of $\Delta^{k}$ is the smallest integer $n(k)$ such that $T_{p}^{n(k)} \Delta^{k}=0$ for all $p$. Nicolas and Serre have recently shown that the growth of $n(k)$ is bounded by $k^{1 / 2}$ but omitted the proof of a key result. We give a new, elementary proof which highlights that the Hecke action is essentially 2-adically continuous in $k$.
\end{abstract}

Keywords: Modular forms modulo 2, Hecke operators, Order of nilpotence

AMS Subject Classification: 11F33, 11F25

\section{Introduction}

The ring of modular forms of level $1 \bmod 2$ is isomorphic to $\mathbb{F}_{2}[\Delta] \subset \mathbb{F}_{2}[[q]]$, where the coefficients of $\Delta$ are the reduction mod 2 of Ramanujan's $\tau$ function. For an odd prime $p$, the Hecke operator $T_{p}$ acts on the ideal $(\Delta) \subset \mathbb{F}_{2}[\Delta]$ by

$$
T_{p}\left(\sum_{n=1}^{\infty} a_{n} q^{n}\right)=\sum_{n=1}^{\infty} a_{p n} q^{n}+a_{n} q^{p n} .
$$

This action is nilpotent. The order of nilpotence $n(k)$ of $\Delta^{k}$ is by definition the smallest positive integer such that for any set of primes $p_{i}$ and any $n_{i}$ with $\sum n_{i}=n(k)$, we have

$$
T_{p_{1}}^{n_{1}} \cdot \ldots \cdot T_{p_{r}}^{n_{r}} \Delta^{k}=0
$$

Section 2 recalls the results of Nicolas-Serre in [1] and [2]. They explicitly compute the $n(k)$ and this allows them to determine the structure of the Hecke algebra. More precisely, they extract from the binary expansion of $k$ a pair of integers $n_{3}(k)$ and $n_{5}(k)$ with the property that $k \mapsto\left[n_{3}(k), n_{5}(k)\right]$ gives a bijection between the odd integers and $\mathbb{N}^{2}$. They then show that

$$
T_{3}^{n_{3}(k)} T_{5}^{n_{5}(k)} \Delta^{k}=\Delta
$$

and that $n(k)=n_{3}(k)+n_{5}(k)+1$. The keystone of their argument is a pair of propositions describing exactly the action of $T_{3}$ and $T_{5}$. In essence, the statement for $T_{3}$ is in the following proposition; there is an analogous statement for $T_{5}$. Here, the height of $k$ is the integer $h(k)=n_{3}(k)+n_{5}(k)$.

(c) 2016 Gerbelli-Gauthier. Open Access This article is distributed under the terms of the Creative Commons Attribution 4.0 International License (http://creativecommons.org/licenses/by/4.0/), which permits unrestricted use, distribution, and reproduction in any medium, provided you give appropriate credit to the original author(s) and the source, provide a link to the Creative Commons license, and indicate if changes were made. 
Theorem 1 ([1], Proposition 4.3, rough form). Let $k$ be an odd integer and let $e$ be the highest exponent of $T_{3}\left(\Delta^{k}\right)$. Then $h(e) \leq h(k)-1$. Moreover, in almost all cases, $n_{3}(e)=$ $n_{3}(k)-1$ and $n_{5}(e)=n_{5}(k)$.

Section 3 recalls the precise definition of $n_{3}(k)$ and $n_{5}(k)$ from [1] and derives some properties. In particular, we introduce the idea of viewing $\left[n_{3}(k), n_{5}(k)\right]$ as a coordinate system for the odd integers, as shown in Fig. 1. The theorem then essentially states that for any odd integer $k$ that does not belong to the leftmost column, $T_{3}$ acts by left translation by one unit.

Letting $k^{\prime}$ be the integer to the left of $k$, it is worth noting that the difference $k-k^{\prime}$ is determined by the congruence class of $k$ modulo a large enough power of 2 . For example, if $k \equiv 3 \bmod 4$ then $k^{\prime}=k-2$. Thus the action of $T_{3}$ can be thought of as being 2 -adically continuous in $k$.

In [1], the "rather long and technical" proof of Proposition 4.3 (our Theorem 1) is omitted. The authors indicate that the proof is by induction via a linear recurrence which they have developed ([1], §3):

$$
T_{3}\left(\Delta^{k}\right)=\Delta T_{3}\left(\Delta^{k-3}\right)+\Delta^{4} T_{3}\left(\Delta^{k-4}\right)
$$

Section 4 gives an original proof of Theorem 1. From Nicolas-Serre's recurrences, we produce new ones involving arbitrarily large powers of 2 :

$$
T_{3}\left(\Delta^{k}\right)=\Delta^{2^{i}} T_{3}\left(\Delta^{k-3 \cdot 2^{i}}\right)+\Delta^{4 \cdot 2^{i}} T_{3}\left(\Delta^{k-4 \cdot 2^{i}}\right) .
$$

These deeper recurrences allow us to derive the statement for $k$ from the statement for the integer $k^{\prime}<k$ that is 2-adically closest to $k$. Precisely, (2) allows us to go from the odd integers contained in a certain set $\mathcal{B}_{1}(i+2)$ of representatives $\bmod 2^{i+2}$ to the next set of representatives $\mathcal{B}_{2}(i+2)$. Figure 2 shows both sets for $i=3$; the recurrences should be viewed as allowing to slide $\mathcal{B}_{2}(i+2)$ onto $\mathcal{B}_{1}(i+2)$ to read off the action of $T_{3}$. An analogous but significantly more laborious proof exists for $T_{5}$.

The ideas introduced here appear applicable to a range of situations beyond level 1 and $p=2$. In (Monsky, P: A hecke algebra attached to mod 2 modular forms of level 3, unpublished), Monsky builds on our methods determines the structure of the Hecke

\begin{tabular}{l|lllllllllll}
7 & 85 & 87 & 93 & 95 & 117 & 119 & 125 & 127 & & \\
6 & 81 & 83 & 89 & 91 & 113 & 115 & 121 & 123 & & & \\
5 & 69 & 71 & 77 & 79 & 101 & 103 & 109 & 111 & & \\
4 & 65 & 67 & 73 & 75 & 97 & 99 & 105 & 107 & & \\
3 & 21 & 23 & 29 & 31 & 53 & 55 & 61 & 63 & & & \\
2 & 17 & 19 & 25 & 27 & 49 & 51 & 57 & 59 & & \\
1 & 5 & 7 & 13 & 15 & 37 & 39 & 45 & 47 & 133 & 135 \\
& 1 & 3 & 9 & 11 & 33 & 35 & 41 & 43 & 129 & 131 & $\ldots$ \\
\hline & 0 & 0 & 1 & 2 & 3 & 4 & 5 & 6 & 7 & 8 & 9
\end{tabular}

Fig. 1 The odd integers in the coordinate system $\left[n_{3}(k), n_{5}(k)\right]$ 


\begin{tabular}{|c|c|c|c|c|c|c|c|c|c|c|}
\hline 7 & 85 & 87 & 93 & 95 & 117 & 119 & 125 & 127 & & \\
\hline 6 & 81 & 83 & 89 & 91 & 113 & 115 & 121 & 123 & & \\
\hline 5 & 69 & 71 & 77 & 79 & 101 & 103 & 109 & 111 & & \\
\hline 4 & 65 & 67 & 73 & 75 & 97 & 99 & 105 & 107 & & \\
\hline 3 & 21 & 23 & 29 & 31 & 53 & 55 & 61 & 63 & & \\
\hline 2 & 17 & 19 & 25 & 27 & 49 & 51 & 57 & 59 & & \\
\hline 1 & 5 & 7 & 13 & 15 & 37 & 39 & 45 & 47 & 133 & 135 \\
\hline 0 & 1 & 3 & 9 & 11 & 33 & 35 & 41 & 43 & 129 & $131 \ldots$ \\
\hline & 0 & 1 & 2 & 3 & 4 & 5 & 6 & 7 & 8 & 9 \\
\hline
\end{tabular}

Fig. 2 The sets $\boldsymbol{B}_{1}$ (5) (in red) and $\boldsymbol{B}_{2}$ (5) (in blue)

algebra attached to modular forms of level 3 modulo 2. Similar "deep recurrences" also appear in Medvedovsky's so-called nilpotence method ([3], § 5) which gives lower bounds on Krull dimensions of Hecke algebras acting on modular forms $\bmod p$. Her initial results hold for all primes $p$ such that $X_{0}(p)$ has genus zero.

\section{Modular forms mod 2: review of the results}

This section gives a brief account of some of the results of [1] and [2] on the Hecke algebra acting on the ring of modular forms mod 2 . The precise definitions of the terms we will use are given in Section 3.

\subsection{The order of nilpotence of a modular form}

The ring of modular forms of level $1 \bmod 2$ is isomorphic to the polynomial ring $\mathbb{F}_{2}[\Delta]$, where $\Delta$ is the formal power series over $\mathbb{F}_{2}$ whose coefficients are the reduction mod 2 of Ramanujan's $\tau$ function. In [1], Nicolas and Serre investigate the action of the Hecke algebra on $(\Delta) \subset \mathbb{F}_{2}[\Delta]$, the space of cusp forms. For an odd prime $p$, the action of $T_{p}$ is given by

$$
T_{p}\left(\sum_{n=1}^{\infty} a_{n} q^{n}\right)=\sum_{n=1}^{\infty} a_{p n} q^{n}+a_{n} q^{p n} .
$$

\section{Example.}

$$
T_{p}(\Delta)=0 \text { for all } p, \quad T_{3}\left(\Delta^{5}\right)=T_{5}\left(\Delta^{3}\right)=0, \quad T_{3}\left(\Delta^{7}\right)=\Delta^{5}, \quad T_{5}\left(\Delta^{7}\right)=\Delta^{3} .
$$

Working mod 2 gives the identity $T_{p}\left(\Delta^{2 k}\right)=\left(T_{p}\left(\Delta^{k}\right)\right)^{2}$. Thus the action of $T_{p}$ on cusp forms mod 2 is determined by its action on the space $\mathcal{F}$ spanned by odd powers of $\Delta$. Following [1], let $\mathcal{A}$ be the subalgebra of $\operatorname{End}(\mathcal{F})$ generated by the $T_{p}$ and let $\mathfrak{m} \subset \mathcal{A}$ be the ideal generated by the $T_{p}$. Nicolas and Serre describe the of the action of $\mathcal{A}$ on $\mathcal{F}$ by computing the order of nilpotence of each $\Delta^{k}$. This is defined as the smallest integer $n(k)$ such that $\mathfrak{m}^{n(k)}\left(\Delta^{k}\right)=0$. In order to do this, they define the code $\left[n_{3}(k), n_{5}(k)\right]$ and the height $h(k)=n_{3}(k)+n_{5}(k)$ of an odd integer $k$. The height essentially measures the complexity of the binary expansion of $k$; it grows like $k^{\frac{1}{2}}$. The surprising main theorem of [1] is that 
$n(k)=h(k)+1$, that is, that the binary expansion of $k$ controls the nilpotence of $\Delta^{k}$. Even more precisely, Niolas-Serre show that the lower bound on nilpotence is achieved by

$$
T_{3}^{n_{3}(k)} T_{5}^{n_{5}(k)} \Delta^{k}=\Delta \text {. }
$$

\subsection{Structure of the Hecke algebra}

In the sequel [2], Nicolas and Serre use the results about the action of $T_{3}$ and $T_{5}$ to determine the structure of the Hecke algebra $\mathcal{A}$ acting on $\mathcal{F}$. For each $n$, let $\mathcal{F}(n)$ be the space spanned by $\Delta, \Delta^{3}, \ldots, \Delta^{2 n+1}$. Let $\mathcal{A}(n)$ be the subalgebra of $\operatorname{End}(\mathcal{F}(n))$ generated by the $T_{p}$. Let $e$ be the element of the dual $\mathcal{F}(n)^{*}$ that maps a polynomial $f(\Delta)$ to the coefficient of $q$ in its Fourier expansion. Nicolas-Serre identify $\mathcal{A}(n)$ with the dual $\mathcal{F}(n)^{*}$ via the map $T_{p} \rightarrow e \circ T_{p}$. Furthermore, they show that there is a surjective morphism

$$
\psi_{n}: \mathbb{F}_{2}[x, y] \rightarrow \mathcal{A}(n), \quad \psi(x)=T_{3}, \quad \psi(y)=T_{5} .
$$

The $\mathcal{A}(n)$ form a projective system under restriction of the action; its inverse limit is the algebra $\mathcal{A}$ of Hecke operators acting of $\mathcal{F}$. In the limit, the morphism $\psi$ becomes injective and the authors conclude that $\mathcal{A} \simeq \mathbb{F}_{2}[[x, y]]$. They conclude by computing explicit examples.

\section{A dyadic ordering on the odd integers}

In [1], Nicolas and Serre attach to an integer $k$ a pair of numbers obtained from its dyadic expansion. This pair will describe the action of $T_{3}$ and $T_{5}$.

\subsection{Definitions}

Let $k$ be an integer, and write the dyadic expansion of $k$ as

$$
k=\sum_{i=0}^{\infty} \beta_{i}(k) 2^{i}, \quad \beta_{i}(k) \in\{0,1\}, \quad \beta_{i}(k)=0 \text { for } i \text { large enough }
$$

Definition 1. The support of $k$ is the set $\mathcal{S}(k)=\left\{2^{i} \mid \beta_{i}(k)=1\right\}$.

Definition 2. (i) The code of $k$ is the following pair of integers $\left[n_{3}(k), n_{5}(k)\right]$ :

$$
\begin{aligned}
& n_{3}(k)=\sum_{i=0}^{\infty} \beta_{2 i+1}(k) 2^{i}=\sum_{\substack{i=1 \\
i \text { odd }}}^{\infty} \beta_{i}(k) 2^{\frac{i-1}{2}} \\
& n_{5}(k)=\sum_{i=0}^{\infty} \beta_{2 i+2}(k) 2^{i}=\sum_{\substack{i=2 \\
i \text { even }}}^{\infty} \beta_{i}(k) 2^{\frac{i-2}{2}} .
\end{aligned}
$$

Following [1], we will denote this by $k \simeq\left[n_{3}(k), n_{5}(k)\right]$.

(ii) The height of $k$ is the integer $h(k)=n_{3}(k)+n_{5}(k)$.

\section{Example.}

$$
1 \simeq[0,0], \quad 3 \simeq[1,0], \quad 5 \simeq[0,1], \quad 27 \simeq[3,2] .
$$




\section{Remark.}

- If $\mathcal{S}(k)$ only contains odd powers of 2 then $n_{3}(k)=h(k)$. Likewise if $\mathcal{S}(k)$ only contains even powers of 2 then $n_{5}(k)=h(k)$.

- If $k$ is odd we have $\left[n_{3}(k), n_{5}(k)\right]=\left[n_{3}(k+1), n_{5}(k+1)\right]$.

- The map $k \mapsto\left[n_{3}(k), n_{5}(k)\right]$ is a bijection between the odd positive integers and $\mathbb{N}^{2}$. This allows of to view $\left[n_{3}(k), n_{5}(k)\right]$ as coordinates in the plane.

Still following [1], we use the code to define a new order relation on $\mathbb{Z}_{\geq 0}$.

Definition 3. If $k$ and $\ell$ are positive integers, we define the relation $\prec$ as follows:

$$
k \prec \ell \text { if }\left\{\begin{array}{l}
h(k)<h(\ell) \text { or } \\
h(k)=h(\ell) \text { and } n_{5}(k)<n_{5}(\ell) .
\end{array}\right.
$$

If $k \prec k^{\prime}$, we say that $k$ is lower than $k^{\prime}$ and that $k^{\prime}$ is higher than $k$.

The relation $\prec$ together with the standard equality becomes a total order when restricted to the odd integers. In terms of Fig. 3, the heights correspond to the diagonals of slope $\nwarrow$, with the arrow pointing towards greater integers. The lowest odd integers are, in order of increasing height, $1,3,5,9,7,17,11,13,19,21 \ldots$.

\subsection{Properties}

We now deduce some properties of the code as elementary consequences of binary arithmetic. The first lemma concerns congruences modulo powers of 2 and follows directly from the definitions of $n_{3}(k)$ and $n_{5}(k)$.

Lemma 1. Let $k, \ell$ be odd integers and for any integer $n$, let $v_{2}(n)$ be the 2-adic valuation of $n$. Then $v_{2}(k-\ell) \geq i$ if and only if

$$
v_{2}\left(n_{3}(k)-n_{3}(\ell)\right) \geq\left\lfloor\frac{i}{2}\right\rfloor \text { and } v_{2}\left(n_{5}(k)-n_{5}(\ell)\right) \geq\left\lfloor\frac{i-1}{2}\right\rfloor,
$$

\begin{tabular}{|c|c|c|c|c|c|c|c|c|c|c|}
\hline 7 & 85 & 87 & 93 & 95 & 117 & 119 & 125 & 127 & & \\
\hline 6 & 81 & 83 & 89 & 91 & 113 & 115 & 121 & 123 & & \\
\hline 5 & 69 & 71 & 77 & 79 & 101 & 103 & 109 & 111 & & \\
\hline 4 & 65 & 67 & 73 & 75 & 97 & 99 & 105 & 107 & & \\
\hline 3 & 21 & 23 & 29 & 31 & 53 & 55 & 61 & 63 & & \\
\hline 2 & 17 & 19 & 25 & 27 & 49 & 51 & 57 & 59 & & \\
\hline 1 & 5 & 7 & 13 & 15 & 37 & 39 & 45 & 47 & 133 & 135 \\
\hline 0 & 1 & 3 & 9 & 11 & 33 & 35 & 41 & 43 & 129 & $131 \ldots$ \\
\hline & 0 & 1 & 2 & 3 & 4 & 5 & 6 & 7 & 8 & 9 \\
\hline
\end{tabular}

where $\lfloor x\rfloor$ is the largest integer smaller than $x$.

Fig. 3 The odd integers in the coordinate system $\left[n_{3}(k), n_{5}(k)\right]$ 
Addition is not order-preserving for $\prec$ but we show in the next lemma that the height of a sum can nevertheless be bounded. We first introduce the notion of a code with negative coefficients in order to account for the fact that adding a positive integer can result in a decrease in the code.

Definition 4. Let $k$ be an integer and let d be a representation of $k$ as a sum of powers of 2

$$
\sum_{i=1}^{\infty} \beta_{i}(d) 2^{i}=k
$$

where this time, $\beta_{i}(d) \in\{-1,0,1\}$. Note that this representation is no longer uniquely determined by $k$.

(i) We define the code of $k$ associated to $d$ as for the standard code:

$$
\begin{aligned}
& n_{3}(k, d)=\sum_{i=0}^{\infty} \beta_{2 i+1}(d) 2^{i}=\sum_{\substack{i=1 \\
i \text { odd }}}^{\infty} \beta_{i}(d) 2^{\frac{i-1}{2}} \\
& n_{5}(k, d)=\sum_{i=0}^{\infty} \beta_{2 i+2}(d) 2^{i}=\sum_{\substack{i=2 \\
i \text { even }}}^{\infty} \beta_{i}(d) 2^{\frac{i-2}{2}} .
\end{aligned}
$$

This is denoted $k \sim\left[n_{3}(k, d), n_{5}(k, d)\right]$.

(ii) For $k$ fixed, the order relation $\prec$ from the previous section is defined on the set of all codes of $k$.

The following lemma borrows heavily from Proposition 2 in Nicolas-Serre's unpublished manuscript detailing their proof of Proposition 4.3.

Lemma 2. Let $k$ and $\ell$ be integers with $\ell$ even.

(i) We have

$$
h(\ell+k) \leq h(\ell)+h(k) .
$$

(ii) If equality is achieved, then $\mathcal{S}(k) \cap \mathcal{S}(\ell)$ only contains even powers of 2 , and if $2^{2 i} \in \mathcal{S}(k) \cap \mathcal{S}(\ell)$, then $2^{2 i+1} \notin \mathcal{S}(k) \cup \mathcal{S}(\ell)$ and moreover we have

$$
n_{5}(k+\ell)=n_{5}(k)+n_{5}(\ell)-\sum_{2^{2 i} \in \mathcal{S}(k) \cap \mathcal{S}(\ell)} 2^{i} .
$$

(iii) In particular if $k$ is odd and $m$ is the odd integer such that

$$
m \simeq\left[n_{3}(k)+n_{3}(\ell), n_{5}(k)+n_{5}(\ell)\right],
$$

we have

$$
k+\ell \preceq m
$$

with equality precisely when $\mathcal{S}(k) \cap \mathcal{S}(l)=\emptyset$.

Proof. Statement (iii) follows from (i) and (ii) and the definition of the order relation $\prec$. We will first prove (i) and (ii) when $\ell=2^{i} \simeq[a, b]$. If $2^{i} \notin \mathcal{S}(k)$, then $\mathcal{S}\left(k+2^{i}\right)=$ $\mathcal{S}(k) \cup\left\{2^{i}\right\}$ so

$$
\left[n_{3}\left(k+2^{i}\right), n_{5}\left(k+2^{i}\right)\right]=\left[n_{3}(k)+a, n_{5}(k)+b\right] .
$$


If $2^{i} \in \mathcal{S}(k)$, consider the set of all possible codes $2^{i} \sim \quad\left[n_{3}\left(2^{i}, d\right), n_{5}\left(2^{i}, d\right)\right]$, allowing negative entries. These come from all possible binary representations of $2^{i}$ with negative coefficients, which are of the form

$$
d_{0}: 2^{i}, \quad d_{1}: 2^{i+1}-2^{i}, \quad d_{2}: 2^{i+2}-2^{i+1}-2^{i}, \quad d_{r}: 2^{i+r}-\sum_{i=0}^{r-1} 2^{i}
$$

One of these representations corresponds to the change in the code of $k$ induced by adding $2^{i}$. Precisely, let $\mathcal{S}^{\prime}=\left\{2^{i}, 2^{i+1}, \ldots, 2^{i+n-1}\right\} \subset \mathcal{S}(k)$ be the longest list of consecutive powers of 2 starting with $2^{i}$ and contained in $\mathcal{S}(k)$. Then doing the addition with carries in binary arithmetic shows that $\mathcal{S}\left(k+2^{i}\right)=\mathcal{S}(k) \cup\left\{2^{i+n}\right\} \backslash \mathcal{S}^{\prime}$. Thus $\left[n_{3}\left(2^{i}, d_{n}\right), n_{5}\left(2^{i}, d_{n}\right)\right]$ represents the change on the code of $k$ resulting from the addition of $2^{i}$. In other words

$$
\left[n_{3}\left(k+2^{i}\right), n_{5}\left(k+2^{i}\right)\right]=\left[n_{3}(k)+n_{3}\left(2^{i}, d_{n}\right), n_{5}(k)+n_{5}\left(2^{i}, d_{n}\right)\right] .
$$

The claim (i) is that in this setting,

$$
h\left(k+2^{i}\right) \leq h(k)+h\left(2^{i}\right)=h(k)+a+b .
$$

Part (ii) states that there is equality if and only if $n=1$ and $i$ is even, in which case $n_{5}(k)+n_{5}\left(2^{i}, d_{n}\right)<n_{5}(k)+b$. By (8), it suffices to show that $n_{3}\left(2^{i}, d_{n}\right)+n_{5}\left(2^{i}, d_{n}\right) \leq a+b$ and that $n_{5}\left(2^{i}, d_{n}\right)<b$ if equality is reached. For this, one only needs to check that the sequence $n_{3}\left(2^{i}, d_{n}\right)+n_{5}\left(2^{i}, d_{n}\right)$ is decreasing with $n$ and that and if $a+b=n_{3}\left(2^{i}, d_{n}\right)+$ $n_{5}\left(2^{i}, d_{n}\right)$ then $i$ is even, $n=1$ and $n_{5}\left(2^{i}, d_{n}\right)=b-2^{\frac{i}{2}}$. Checking this requires only the definition of the code.

When $\ell \neq 2^{i}$, (i) holds if one successively adds all powers of 2 contained in $\mathcal{S}(\ell)$. Likewise, (ii) holds inductively, since in order to preserve equality at each step, $2^{i} \in \mathcal{S}(\ell)$ must satisfy that $i$ is even and $2^{i+1} \notin \mathcal{S}(k)$.

Corollary 1. If $0<k<2^{i}$, then $h\left(k+2^{i}\right)=h(k)+h\left(2^{i}\right)$.

\subsection{The code of a polynomial}

We extend the definition of the code to certain polynomials. Recall that $\mathcal{F} \subset \mathbb{F}_{2}[\Delta]$ is the subspace of polynomials with odd exponents.

Definition 5. Let $f \in \mathcal{F}$. Then $f$ can be written as

$$
f=\Delta^{e_{1}}+\ldots+\Delta^{e_{n}}, \quad e_{1} \succ \ldots \succ e_{n} .
$$

We define the code of $f$ to be the code of its highest exponent: $\left[n_{3}(f), n_{5}(f)\right]=$ $\left[n_{3}\left(e_{1}\right), n_{5}\left(e_{1}\right)\right]$. The height $h(f)$ and support $\mathcal{S}(f)$ are likewise defined as $h\left(e_{1}\right)$ and $\mathcal{S}\left(e_{1}\right)$.

Lemma 3. As an immediate consequence, iff, $g \in \mathcal{F}$ then

$$
h(f+g) \leq \max \{h(f), h(g)\} .
$$


Lemma 4. Let $f \in \mathcal{F}$. Then $h\left(\Delta^{2^{i}} f\right) \leq h(f)+h\left(2^{i}\right)$. If equality is reached, then one of the following holds:

(i) $2^{i} \notin \mathcal{S}(f)$,

(ii) $i$ is even, $2^{i} \in \mathcal{S}(f)$ but $2^{i+1}$ is not. In this case,

$$
\left[n_{3}\left(\Delta^{2^{i}} f\right), n_{5}\left(\Delta^{2^{i}} f\right)\right]=\left[n_{3}(f)+2^{\frac{i}{2}}, n_{5}(f)-2^{\frac{i}{2}}\right] .
$$

Proof. Let $k$ be the highest exponent of $f$. If $k+2^{i}$ is the highest exponent of $\Delta^{2} f$, then the statement is an immediate consequence of Lemma 2. Otherwise there is another exponent $j \prec k$ such that $j+2^{i} \succ k+2^{i}$. In that case Lemma 2 still gives the bounds $h\left(j+2^{i}\right) \leq h(j)+h\left(2^{i}\right) \leq h(k)+h\left(2^{i}\right)$.

\section{The action of $T_{3}$}

\subsection{The recurrences}

Recall that $\mathcal{F}$ is the subspace of $\mathbb{F}_{2}[\Delta]$ spanned by odd powers of $\Delta$. In [1], Nicolas and Serre determine the following recurrence formula for the action of $T_{3}$ on $\mathcal{F}$. Suppose $k \geq 4$, then

$$
T_{3}\left(\Delta^{k}\right)=\Delta T_{3}\left(\Delta^{k-3}\right)+\Delta^{4} T_{3}\left(\Delta^{k-4}\right) .
$$

This recursive linear relation for the action of $T_{3}$ is the central tool for our proof. We first produce from it what we refer to as deeper recurrences.

Lemma 5. For all $i \geq 0$, if $k \geq 2^{i+2}$, then $T_{3}\left(\Delta^{k}\right)$ satisfies the following relation:

$$
T_{3}\left(\Delta^{k}\right)=\Delta^{2^{i}} T_{3}\left(\Delta^{k-3 \cdot 2^{i}}\right)+\Delta^{4 \cdot 2^{i}} T_{3}\left(\Delta^{k-4 \cdot 2^{i}}\right) .
$$

Proof. The proof is by induction on $i$ with starting point (9). We get the $i+1^{\text {th }}$ identity by composing the $i^{\text {th }}$ identity with itself; repeated terms cancel since we work modulo 2 .

\subsection{Notation}

Let $k \simeq[a, b]$ be odd. We will interchangeably use $k$ and $[a, b]$, and unless specified otherwise, $[a, b]$ will always denote the odd integer with the prescribed code. Additionally, let $T_{[a, b]}$ denote $T_{3}\left(\Delta^{k}\right)$. Similarly, let $T_{[a, b]-2^{i+2}}$ denote $T_{3}\left(\Delta^{k-2^{i+2}}\right)$ and $T_{[a, b]-3 \cdot 2^{i}}$ denote $T_{3}\left(\Delta^{k-3 \cdot 2^{i}}\right)$.

\subsection{Proof of the theorem}

Proposition 4.3 in [1] states that the coordinate $n_{3}(k)$ encodes the action of $T_{3}$. It, together with the analogous proposition for $T_{5}$, constitutes the key technical result of [1].

Theorem 1 (Proposition 4.3, [1]). Let $f \in \mathcal{F}$, let $[a, b]$ be the highest exponent off, and let $[c, d]$ denote the highest exponent of $T_{3}(f)$. Then:

(i) $c+d \leq a+b-1$

(ii) If $a \neq 0$, then $[c, d]=[a-1, b]$.

Lemma 6. If Theorem 1 holds for monomials in $\mathcal{F}$ then it holds for all $f \in \mathcal{F}$. 
Proof. Assume the theorem holds for monomials and let $f \in \mathcal{F}$. It can be written:

$$
f=\Delta^{e_{1}}+\ldots+\Delta^{e_{n}}, \quad e_{1} \succ \ldots \succ e_{n}, \quad e_{i} \simeq\left[a_{i}, b_{i}\right] .
$$

We now apply $T_{3}$ and get that:

$$
T_{3}(f)=T_{\left[a_{1}, b_{1}\right]}+T_{\left[a_{2}, b_{2}\right]}+\ldots+T_{\left[a_{n}, b_{n}\right]} .
$$

Let $\left[c_{i}, d_{i}\right]$ be the highest exponent of $T_{\left[a_{i}, b_{i}\right]}$. If $a_{1} \neq 0$ then $\left[c_{1}, d_{1}\right]=\left[a_{1}-1, b_{1}\right]$. For all $i>1$, we have

$$
c_{i}+d_{i} \leq a_{i}+b_{i}-1 \leq a_{1}+b_{1}-1 .
$$

If both inequalities are equalities, then $a_{i}+b_{i}=a_{1}+b_{1}$, which implies that $b_{i}<b_{1}$ since $e_{1}$ is the highest term. Thus $\left[c_{i}, d_{i}\right] \prec\left[a_{1}-1, b_{1}\right]$ and the theorem holds. If $a_{1}=0$, all we need to show is that $c_{i}+d_{i} \leq b_{1}-1$ for all $i$. This follows immediately from the statement for monomials since $a_{i}+b_{i} \leq b_{1}$ by definition of $\prec$.

Proof of Theorem 1. The proof is by induction on $k$, using the recurrence of Lemma 5:

$$
T_{[a, b]}=\Delta^{2^{i}} T_{[a, b]-3 \cdot 2^{i}}+\Delta^{4 \cdot 2^{i}} T_{[a, b]-4 \cdot 2^{i}}
$$

This formula will allow us to recover $T_{3}\left(\Delta^{k}\right)$ from $T_{3}\left(\Delta^{k^{\prime}}\right)$ where $k^{\prime}<k$ and $v_{2}\left(k-k^{\prime}\right)$ is large. The proof is split in three cases. Case 0 is concerned with integers $k \simeq[a, b]$ where $a=0$; in this case only (i) holds. Case 1 could be deemed the normal situation: induction is straightforward and the highest term will appear in $\Delta^{4 \cdot 2^{i}} T_{[a, b]-4 \cdot 2^{i}}$. The setup for the remaining integers is described in Case 2 .

Case 0: $k \simeq[0, b]$

Let $i$ be such that $2^{i}<b<2^{i+1}$. Then $[0, b]>2^{2 i+2}$ and we use the $2^{2 i^{\text {th }}}$ iteration of the recurrence:

$$
T_{[0, b]}=\Delta^{2^{2 i}} T_{[0, b]-3 \cdot 2^{2 i}}+\Delta^{2^{2 i+2}} T_{[0, b]-2^{2 i+2}} .
$$

By Lemmas 3 and 4, we have

$$
\begin{aligned}
h\left(T_{[0, b]}\right) & \leq \max \left\{h\left(\Delta^{2^{2 i}} T_{[0, b]-3 \cdot 2^{2 i}}\right), h\left(\Delta^{2^{2 i+2}} T_{[0, b]-2^{2 i+2}}\right)\right\} \\
& \leq \max \left\{2^{i-1}+h\left(T_{[0, b]-3 \cdot 2^{2 i}}\right), 2^{i}+h\left(T_{[0, b]-2^{2 i+2}}\right)\right\} .
\end{aligned}
$$

Thus it suffices to bound these two terms. We first compute $\left(T_{[0, b]-2^{2 i+2}}\right)$. Since $2^{2 i+2} \in$ $\mathcal{S}([0, b])$, then $2^{2 i+2} \notin \mathcal{S}\left([0, b]-2^{2 i+2}\right)$ the corollary to Lemma 2 gives

$$
h\left([0, b]-2^{2 i+2}\right)=h([0, b])-h\left(2^{2 i+2}\right)=b-2^{i} .
$$

We apply the induction hypothesis to $T_{[0, b]-2^{2 i+2}}$ and find that

$$
h\left(T_{[0, b]-2^{2 i+2}}\right) \leq h\left([0, b]-2^{2 i+2}\right)-1=b-2^{i}-1 .
$$

It follows that $2^{i}+h\left(T_{[0, b]-2^{2 i+2}}\right) \leq b-1$.

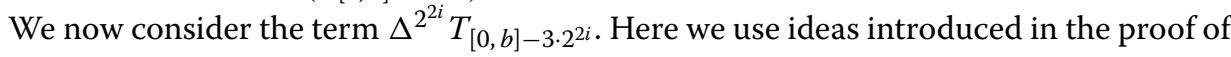
Lemma 2 . All the possible ways in which subtracting $3 \cdot 2^{2 i}$ from $[0, b]$ could modify the code correspond to all admissible dyadic representations of the negative integer $-3 \cdot 2^{2 i}$, where one allows both positive and negative binary coefficients. A quick study of the binary expansion of $b$ reveals two possible representations :

$$
d_{1}:-2^{2 i+2}+2^{2 i+1}-2^{2 i} \sim\left[2^{i},-2^{i}-2^{i-1}\right] \quad d_{2}:-2^{2 i+2}+2^{2 i} \sim\left[0,-2^{i-1}\right] .
$$


This gives two possible cases:

$$
\Delta^{2^{2 i}} T_{[0, b]-3 \cdot 2^{2 i}}=\Delta^{2^{2 i}} T_{\left[2^{i}, b-2^{i}-2^{i-1}\right]} \quad \text { or } \quad \Delta^{2^{2 i}} T_{[0, b]-3 \cdot 2^{2 i}}=\Delta^{2^{2 i}} T_{\left[0, b-2^{i-1}\right]}
$$

We apply the induction hypothesis and use Lemma 4:

$$
\begin{aligned}
& h\left(T_{\left[2^{i}, b-2^{i}-2^{i-1}\right]}\right)=b-1+\left(2^{i}-2^{i}\right)-2^{i-1} \Rightarrow h\left(\Delta^{2^{2 i}} T_{\left[2^{i}, b-2^{i}-2^{i-1}\right]}\right) \leq b-1 \\
& h\left(T_{\left[0, b-2^{i-1}\right]}\right) \leq b-2^{i-1}-1 \quad \Rightarrow \quad h\left(\Delta^{2^{2 i}} T_{\left[0, b-2^{i-1}\right]}\right) \leq b-1 .
\end{aligned}
$$

This shows that all terms in the recurrence have height less than $b-1$.

\section{Case $1: k \simeq[a, b]$ where $a \neq 0$ is not a single, maximal power of 2 .}

Let $i$ be the integer such that $2^{i+2}<[a, b]<2^{i+3}$, and assume that $a \neq 2^{\frac{i+1}{2}}$. We will explain the induction process using the table introduced in Fig 3. Let

$$
\mathcal{B}_{n}(i)=\left\{k \in \mathbb{Z}_{\text {odd }} \mid(n-1) \cdot 2^{i}<k<n \cdot 2^{i}\right\}
$$

We refer to the $\mathcal{B}_{n}(i)$ as boxes. When $\left[n_{3}(k), n_{5}(k)\right]$ are used as a coordinate system in the plane, these subsets are indeed rectangular: it follows from Lemma 1 that they have dimensions $2^{\left\lfloor\frac{i}{2}\right\rfloor} \times 2^{\left\lfloor\frac{i-1}{2}\right\rfloor}$. For fixed $i$, the $\left\{\mathcal{B}_{n}(i)\right\}_{n \in \mathbb{N}}$ partition the odd integers; each $\mathcal{B}_{n}(i)$ contains a set of representatives for all odd residue classes mod $2^{i}$. For an example, see Fig 4. Since all boxes have the same shape and size, it is natural to compare the relative positions of integers in 2 different boxes. Lemma 1 ensures that the relative positions have the following key property.

Key Property The odd integers $\ell \in \mathcal{B}_{n}(i)$ and $\ell+2^{i} \in \mathcal{B}_{n+1}(i)$ occupy the same relative positions inside their respective boxes.

By construction, $[a, b] \in \mathcal{B}_{2}(i+2)$. Assume by induction that Theorem 1 holds for all

\begin{tabular}{|c|c|c|c|c|c|c|c|c|c|c|c|}
\hline 7 & 85 & 87 & 93 & 95 & 117 & 119 & 125 & 127 & & & \\
\hline 6 & 81 & 83 & 89 & 91 & 113 & 115 & 121 & 123 & & & \\
\hline 5 & 69 & 71 & 77 & 79 & 101 & 103 & 109 & 111 & & & \\
\hline 4 & 65 & 67 & 73 & 75 & 97 & 99 & 105 & 107 & & & \\
\hline 3 & 21 & 23 & 29 & 31 & 53 & 55 & 61 & 63 & & & \\
\hline 2 & 17 & 19 & 25 & 27 & 49 & 51 & 57 & 59 & & & \\
\hline 1 & 5 & 7 & 13 & 15 & 37 & 39 & 45 & 47 & 133 & 135 & \\
\hline 0 & 1 & 3 & 9 & 11 & 33 & 35 & 41 & 43 & 129 & 131 & $\ldots$ \\
\hline & 0 & 1 & 2 & 3 & 4 & 5 & 6 & 7 & 8 & 9 & \\
\hline
\end{tabular}
monomials with exponents in $\mathcal{B}_{1}(i+2)$ and let $\left[a^{\prime}, b^{\prime}\right]=[a, b]-2^{i+2}$; by the key property, $\left[a^{\prime}, b^{\prime}\right]$ occupies the same position in $\mathcal{B}_{1}(i+2)$ as $[a, b]$ in $\mathcal{B}_{2}(i+2)$. The restriction on $a$ ensures $a^{\prime} \neq 0$, so by induction, the highest term of $T_{[a, b]-2^{i+2}}$ is $\Delta^{\left[a^{\prime}-1, b^{\prime}\right]}$. Since

Fig. 4 The two boxes $\boldsymbol{B}_{1}$ (5) (in red) and $\boldsymbol{B}_{2}$ (5) (in blue). Observe that integers that differ by $2^{5}$ occupy the same relative position 
$\left[a^{\prime}-1, b^{\prime}\right]$ is the integer directly to the left of $\left[a^{\prime}, b^{\prime}\right]$, using the key property again to from $\mathcal{B}_{1}(i+2)$ to $\mathcal{B}_{2}(i+2)$ gives

$$
\left[a^{\prime}-1, b^{\prime}\right]+2^{i+2}=[a-1, b]
$$

Let $\left[c^{\prime}, d^{\prime}\right]$ be any other exponent of $T_{\left[a^{\prime}, b^{\prime}\right]}$. Then by induction, $\left[c^{\prime}, d^{\prime}\right] \prec\left[a^{\prime}-1, b^{\prime}\right]$. A similar application of the key property shows that $\left[c^{\prime}, d^{\prime}\right]+2^{i+2} \prec[a-1, b]$ and that $[a-1, b]$ is the leading exponent in $\Delta^{2^{i+2}} T_{[a, b]-2^{i+2} \text {. }}$

To complete Case 1 , it now suffices to that the exponents in $\Delta^{2^{i}} T_{[a, b]-3 \cdot 2^{i}}$ are strictly lower than $[a-1, b]$. For this, we pass to smaller boxes $\mathcal{B}_{k} i, 1 \leq k \leq 8$. By construction, $[a, b] \in \mathcal{B}_{k} i$ for $5 \leq k \leq 8$. Let $\left[a^{\prime \prime}, b^{\prime \prime}\right]=[a, b]-3 \cdot 2^{i}$; its code depends on the value of $n$ and the parity of $i$, the latter determining the positions of the $\mathcal{B}_{n} i$ on the grid of odd integers. That is, the shape and arrangement of the boxes are determined by the parity of $i$, and only their size depends on the actual size of $i$.

Case 1A: $i$ is even. If $i$ is even, the boxes are as in Fig. 5 and we compute:

$$
\begin{aligned}
{[a, b] \in \mathcal{B}_{5}(i) \text { or } \mathcal{B}_{7}(i) } & \Rightarrow\left[a^{\prime \prime}, b^{\prime \prime}\right]=\left[a, b-3 \cdot 2^{\frac{i-2}{2}}\right] \\
{[a, b] \in \mathcal{B}_{6}(i) } & \Rightarrow\left[a^{\prime \prime}, b^{\prime \prime}\right]=\left[a+2^{\frac{i}{2}}, b-3 \cdot 2^{\frac{i-2}{2}}\right] \\
{[a, b] \in \mathcal{B}_{8}(i) } & \Rightarrow\left[a^{\prime \prime}, b^{\prime \prime}\right]=\left[a-2^{\frac{i}{2}}, b-2^{\frac{i-2}{2}}\right] .
\end{aligned}
$$

By induction, any exponent $\left[c^{\prime \prime}, d^{\prime \prime}\right]$ of $T_{\left[a^{\prime \prime}, b^{\prime \prime}\right]}$ is lower than $\left[a^{\prime \prime}-1, b^{\prime \prime}\right]$. By Lemma 2 (iii),

$$
[c, d]=\left[c^{\prime \prime}, d^{\prime \prime}\right]+2^{i} \prec\left[c^{\prime \prime}, d^{\prime \prime}+2^{\frac{i-2}{2}}\right] .
$$

By comparing this maximal possible increase with each of the codes of $\left[a^{\prime \prime}, b^{\prime \prime}\right]$ in Eqs. (11)-(13), we find that $[c, d] \prec[a-1, b]$.

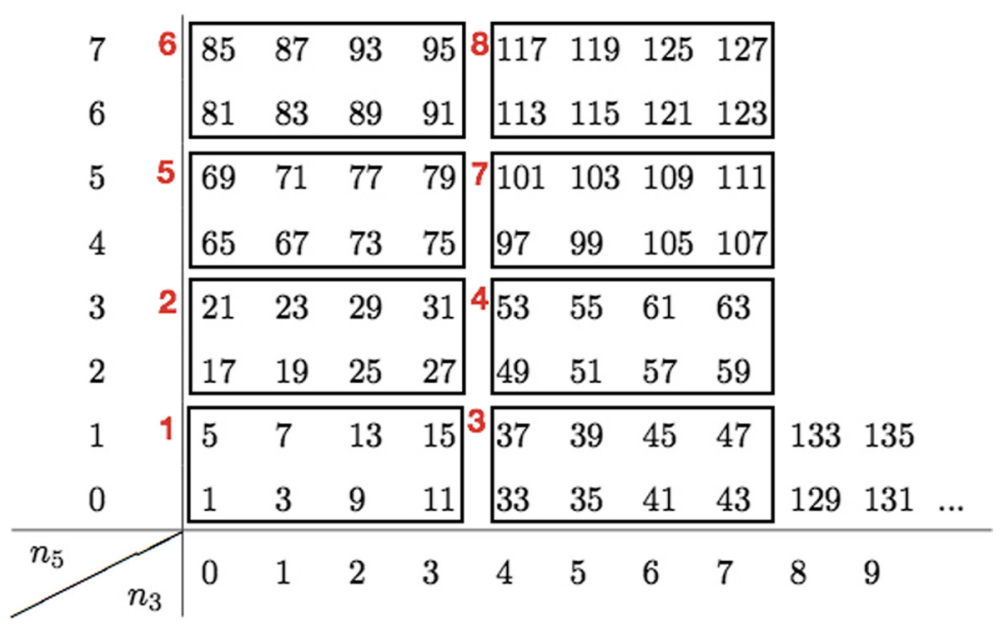

Fig. 5 An example of a partition into 8 boxes for $i$ even (here $i=4$ ) 
Case 1B: $i$ is odd. If $i$ is odd, the $\mathcal{B}_{n}(i)$ are squares of side $2^{\frac{i-1}{2}}$ and arranged as in Fig 6 . Let $[a, b] \in \mathcal{B}_{n}(i)$. Then $\left[a^{\prime \prime}, b^{\prime \prime}\right] \in \mathcal{B}_{n-3}(i)$ and the possible codes are:

$$
\left[a^{\prime \prime}, b^{\prime \prime}\right]= \begin{cases}{\left[a-2^{\frac{i+1}{2}}+2^{\frac{i-1}{2}}, b\right]} & n=5,7 \\ {\left[a-3 \cdot 2^{\frac{i-1}{2}}, b+2^{\frac{i-1}{2}}\right]} & n=6 \\ {\left[a-2^{\frac{i-1}{2}}, b-2^{\frac{i-1}{2}}\right]} & n=8 .\end{cases}
$$

Here, by induction, any exponent $\left[c^{\prime \prime}, d^{\prime \prime}\right]$ of $T_{\left[a^{\prime \prime}, b^{\prime \prime}\right]}$ satisfies $\left[c^{\prime \prime}, d^{\prime \prime}\right] \preceq\left[a^{\prime \prime}-1, b^{\prime \prime}\right]$ and by Lemma 2 (iii):

$$
[c, d]:=\left[c^{\prime \prime}, d^{\prime \prime}\right]+2^{i} \preceq\left[c^{\prime \prime}+2^{\frac{i-1}{2}}, d^{\prime \prime}\right] .
$$

The comparison of (14) and (15) shows that if $n=6,8$ then $c+d<a+b-1$ and all exponents coming from $\Delta^{2^{i}} T_{\left[a^{\prime \prime} b^{\prime \prime}\right]}$ are strictly lower than $[a-1, b]$. However, if $n=5,7$, $\left[c^{\prime \prime}, d^{\prime \prime}\right]=\left[a^{\prime \prime}-1, b^{\prime \prime}\right]$, and equality is reached in (15) for $\left[c^{\prime \prime}, d^{\prime \prime}\right]=\left[a^{\prime \prime}-1, b^{\prime \prime}\right]$, then $c+d=$ $a+b-1$. We will show that these three conditions cannot be fulfilled simultaneously with the restriction that we imposed on $a$.

Assume for contradiction that $n=5,7$ and equality is reached in (15) for $\left[a^{\prime \prime}-1, b^{\prime \prime}\right]$. The condition on the value of $n$ forces $\left[a^{\prime \prime}, b^{\prime \prime}\right] \in \mathcal{B}_{i}(n-3)$ where $n-3=2,4$. Additionally, Lemma 2, part (iii) shows that the second condition is equivalent to $2^{i} \notin \mathcal{S}\left(\left[a^{\prime \prime}-1, b^{\prime \prime}\right]\right)$, which by definition of the $\mathcal{B}_{i}(k)$ implies that $\left[a^{\prime \prime}-1, b^{\prime \prime}\right] \in \mathcal{B}_{m}(i)$ for $m$ odd. Since $\left[a^{\prime \prime}-1, b^{\prime \prime}\right]$ is immediately to the left of $\left[a^{\prime \prime}, b^{\prime \prime}\right]$ in the table, this implies that $\left[a^{\prime \prime}-1, b^{\prime \prime}\right]$ is in the rightmost column of $\mathcal{B}_{n-4}(i)$ and that $\left[a^{\prime \prime}, b^{\prime \prime}\right]$ is in the leftmost column of $\mathcal{B}_{n-3}(i)$. In this case, the key property implies that $[a, b]$ is in the leftmost column of $\mathcal{B}_{n} i$ for $n=5,7$, i.e. that $a=2^{\frac{i+1}{2}}$. This is precisely the case that we exclude by assumption. Thus $[a-1, b]$ does not appear as an exponent of $\Delta^{2^{i}} T_{\left[a^{\prime \prime} b^{\prime \prime}\right]}$ if $a$ is not a single, maximal power of 2 and the proof of this case is complete.

Case $2: 2^{i+2}<[a, b]<2^{i+3}$ and $a=2^{\frac{i+1}{2}}$.

An immediate upshot of the above discussion is that if $a=2^{\frac{i+1}{2}}$, the term $[a-1, b]$ does indeed appear as the leading exponent of $\Delta^{2^{i}} T_{[a, b]-3 \cdot 2^{i}}$.

Thus the situation is the inverse of Case 1: it remains to show that all the exponents of $\Delta^{2^{i+2}} T_{[a, b]-2^{i+2}}=\Delta^{2^{i+2}} T_{[0, b]}$ are strictly lower than $[a-1, b]$. Let $\left[c^{\prime}, d^{\prime}\right]$ be any exponent

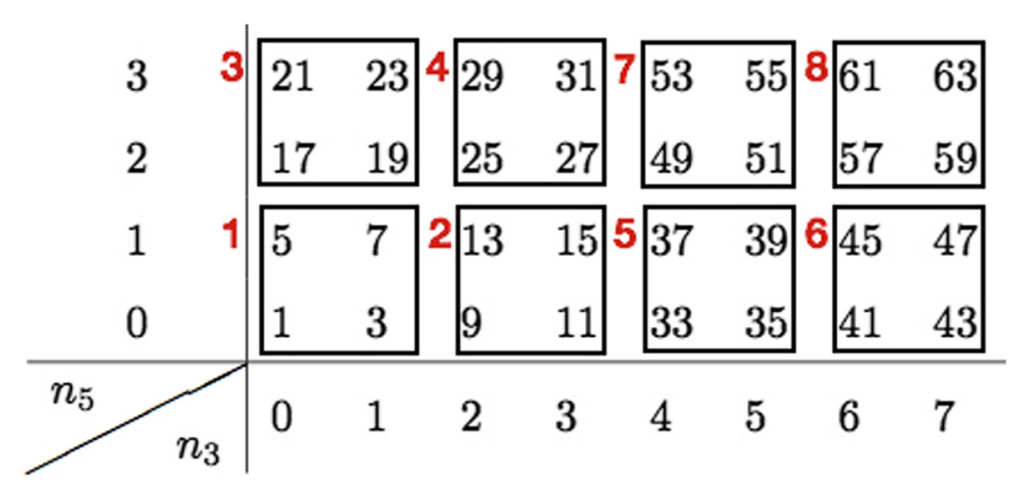

Fig. 6 The arrangement of boxes for $i$ odd (here $i=3$ ) 
in $T_{[0, b]}$, and $[c, d]=\left[c^{\prime}, d^{\prime}\right]+2^{i+2}$. Then by the induction hypothesis and Lemma 2 ,

$$
c^{\prime}+d^{\prime} \leq b-1 \Rightarrow c+d \leq 2^{\frac{i+1}{2}}+b-1=a+b-1 .
$$

This could still be an equality. But note that $[0, b-1]<[0, b]<2^{i+2}$ and that $[0, b-1]$ is the largest integer with height $b-1$, so $\left[c^{\prime}, d^{\prime}\right]<2^{i+2}$. It then follows from Corollary 1 that

$$
[c, d]=\left[c^{\prime}, d^{\prime}\right]+2^{\frac{i+2}{2}}=\left[c^{\prime}+2^{\frac{i+1}{2}}, d^{\prime}\right] .
$$

We find that $d \leq b-1$ and $[c, d] \prec[a-1, b]$, which completes the case and concludes the proof.

\section{Competing interests}

The author declares that she has no competing interests.

\section{Acknowledgements}

The author is grateful for Henri Darmon's excellent and dedicated advising. Her gratitude goes to Jean-Louis Nicolas and Jean-Pierre Serre for their generous attention and providing their version of the proof. She is thankful to Paul Monsky for important suggestions in the presentation and to Olivier Martin for fruitful exchanges. This research was conducted when the author was supported by a NSERC SURA scholarship. The author thanks the anonymous referees for their comments.

Received: 14 January 2016 Accepted: 15 January 2016

Published online: 14 March 2016

\section{References}

1. Nicolas, J-L, Serre, J-P: Formes modulaires modulo 2: Ordre de nilpotence des opérateurs de hecke. C.R. Acad. Sci. Paris. 350(7-8), 343-348 (2012)

2. Nicolas, J-L, Serre, J-P: Formes modulaires modulo 2: Structure de l'algèbre de hecke. C.R. Acad. Sci. Paris. 350(9-10), 449-454 (2012)

3. Medvedovsky, A: Lower bounds on the dimension of mod- $p$ hecke algebras: the nilpotence method. PhD thesis, Brandeis University, Department of Mathematics, Watham, MA (2015)

\section{Submit your manuscript to a SpringerOpen ${ }^{\circ}$ journal and benefit from:}

- Convenient online submission

- Rigorous peer review

- Immediate publication on acceptance

- Open access: articles freely available online

- High visibility within the field

- Retaining the copyright to your article 